\title{
USING PARAMETERIZATION OF OBJECTS IN AUTODESK INVENTOR IN DESIGNING STRUCTURAL CONNECTORS
}

\author{
Gabriel Borowski', Agnieszka Jankowska², Magdalena Paśnikowska² \\ 1 Faculty of Fundamentals of Technology, Lublin University of Technology, Nadbystrzycka 38 Str., 20-618 \\ Lublin, Poland, e-mail: g.borowski@pollub.pl \\ 2 Student of the Faculty of Fundamentals of Technology, Lublin University of Technology, Nadbystrzycka 38 \\ Str., 20-618 Lublin, Poland, e-mail: aga.jankowska89@wp.pl; pasnikowskamagdalena@gmail.com
}

Received: 2015.04.30

Accepted: 2015.05.15

Published: 2015.06.01

\begin{abstract}
The article presents the parameterization of objects used for designing the type of elements as structural connectors and making modifications of their characteristics. The design process was carried out using Autodesk Inventor 2015. We show the latest software tools, which were used for parameterization and modeling selected types of structural connectors. We also show examples of the use of parameterization facilities in the process of constructing some details and making changes to geometry with holding of the shape the element. The presented method of Inventor usage has enabled fast and efficient creation of new objects based on sketches created.
\end{abstract}

Keywords: parameterization, designing, structural connectors.

\section{INTRODUCTION}

The development of computer-aided design and manufacturing processes makes it possible to quickly design products that can be tested before they are put into production. As a result, the design process is becoming cheaper [1]. To further simplify the design process is the introduction of the parameterization of objects [6]. Parameterization of objects in the design process is subject to providing the bonds of cohesion and dimensional bonds, whose modification adjusts the characteristics of the object [3].

In a typical design process, in addition to conducting activities related to 3D modeling and editing of 2D documentation, it shall also be made to modify the project [10]. Implementation of the proposed modifications is particularly important when we produce goods in small production series and we improve it cyclically. In most CAD systems, including Autodesk Inventor package, the solution to this problem is the appropriate use of parameterization mechanisms. Parametric model guarantees automatically modifying, as a result of changes in predefined attributes, for example dimensions. This is done by well-defined mathematical functions and relationships through which they are referred to objects of type sketch (dimensional constraints, geometric constraints), the elements (the depth of element) and the relative positions of the components in unit (mounting ties) [10].

Parameterization in the designing is most commonly used for programming the production process of parts with a similar geometric structure differing characteristic dimensions. Parameterization process also used for programming and "automation" CNC machines with Heidenhain TNC620 control system [8]. It has been found that this type of parameterization allows to shorten the preparation time of the technological process, especially when the parts are machined with similar geometric shapes, characterized by a variability in the dimensions of some elements of the object.

The goal of article is to show how to use parameterization of objects in designing structural connectors using Autodesk Inventor 2015. 


\section{CREATING PARAMETRIC OBJECTS USING CAD SOFTWARE}

Creating a parametric object begins with a two-dimensional drawing to which the CAD user later applies appropriate geometric constraints [7]. Autodesk Inventor utilises perpendicular and parallel constraints, as well as horizontal and vertical fix of the object [11]. These tools ensure that the designed element's shape will not change once new dimensions are introduced. Constraints are applied hierarchically. Incorrect selection of two edges may lead to misapplication of constraints on the drawing of the designed object.

To start drawing, a suitable tool should be selected, e.g. a line, arc or circle. Notably, if "rectangle" is selected, a suitable parallel and perpendicular constrains are automatically applied to the corresponding pairs of edges. The benefit of designing with the use of parameterisation tool is the ability to freely edit and modify the drawing on every stage of design [9]. Once the desired 2D drawing has been prepared, all necessary details can be added as a basis for generating the complete 3D model [5]. A 3D object may be created from one or more $2 \mathrm{D}$ drawings. The location of particular details can be changed at any time using the Dimensioning tool. Once the three-dimensional model of the element has been generated, it is possible to return to and edit the original drawing, and the generated 3D shape will be changed accordingly [2]. It is important to ensure that the drawing utilises appropriate layers which can be edited if a change in the designed object's dimensions is needed. The

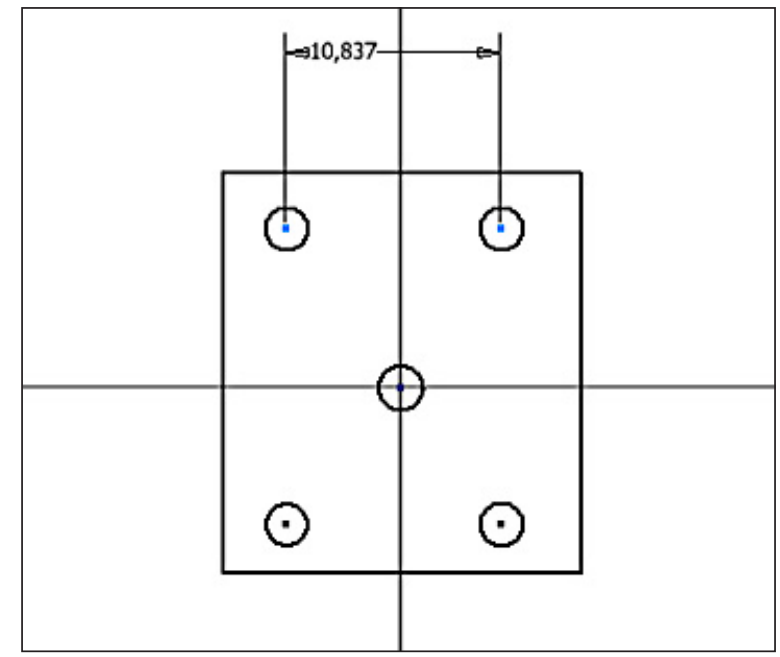

Fig. 1. Drawing a side wall of the connector model and placing holes applied geometric constrains will ensure that the process of updating the object will change its dimensions and introduce additional details, but will not affect the element's actual shape. Geometric models created from a drawing also allow a stress analysis of the element for programmable loads [4].

\section{THE PROCESS OF DESIGNING STRUCTURAL CONNECTORS}

Autodesk Inventor 2015 was used to design a standard structural element - structural connectors. Such elements are commonly used in various industries, most commonly as supporting elements for wooden and steel structures. It is important that the connector always rests flush against the connected elements. It should also be made of a material resistant to weather conditions. Most types of structural connectors have been catalogued and described by applicable norms, but in certain cases a customized connector is needed for a particular structure where e.g. the bolt hole locations must be changed.

The process of designing a connector starts with drawing one wall and placing the holes properly (Fig. 1). The thickness of the connector wall is set using the Stretch command (Fig. 2). Figure 3 illustrates the use of the same command to adjust the position of holes in the connector's side walls. The method of designing the wall of a two-sided connector is presented in Figure 4. The subsequent illustration presets the finished model of a two-sided connector (Fig. 5).

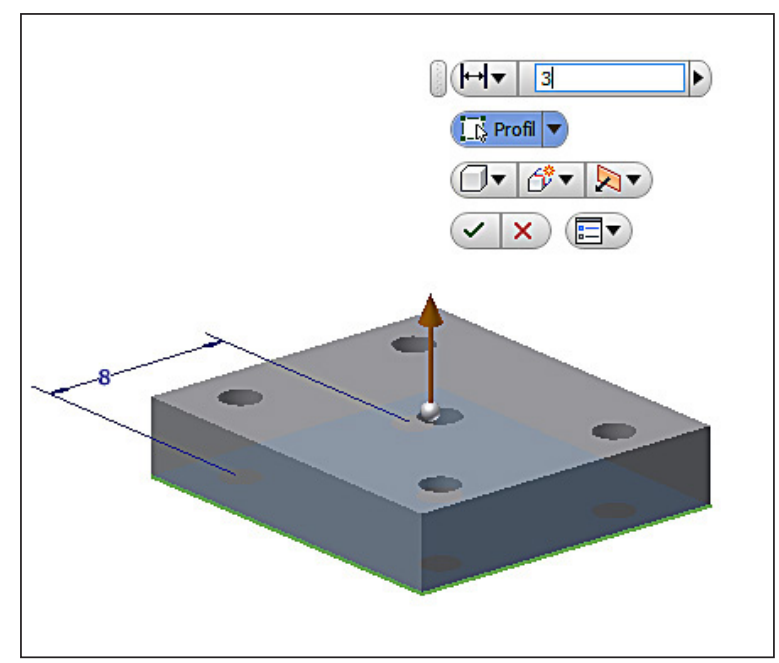

Fig. 2. Using the Stretch function to change the wall thickness 
The method of object parameterisation offered by the Inventor software can also be used in designing other types of structural connectors (Figs. 6, 7 and 8). Figure 6 illustrates modification of hole locations on the model of a two-sided connector. The rearrangement and re-dimensioning of the holes is accomplished by imputing new data into the original drawing by using the Edit function. The program automatically updates the drawing allowing the designer to directly control the effects of the changes being introduced.

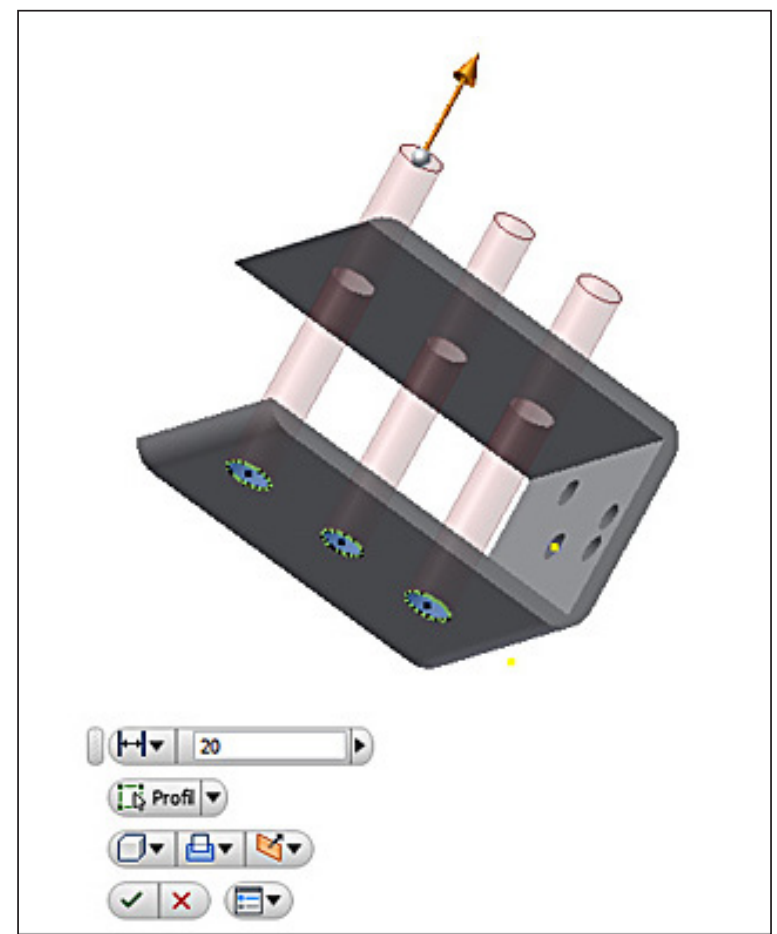

Fig. 3. Using the Stretch function to change location of the holes

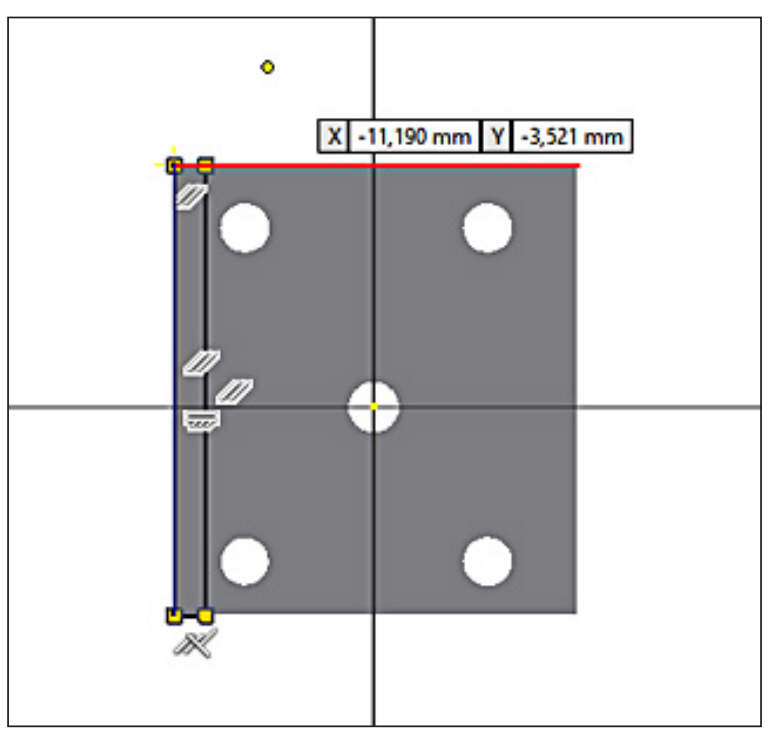

Fig. 4. Designing the side wall of a two-sided connector

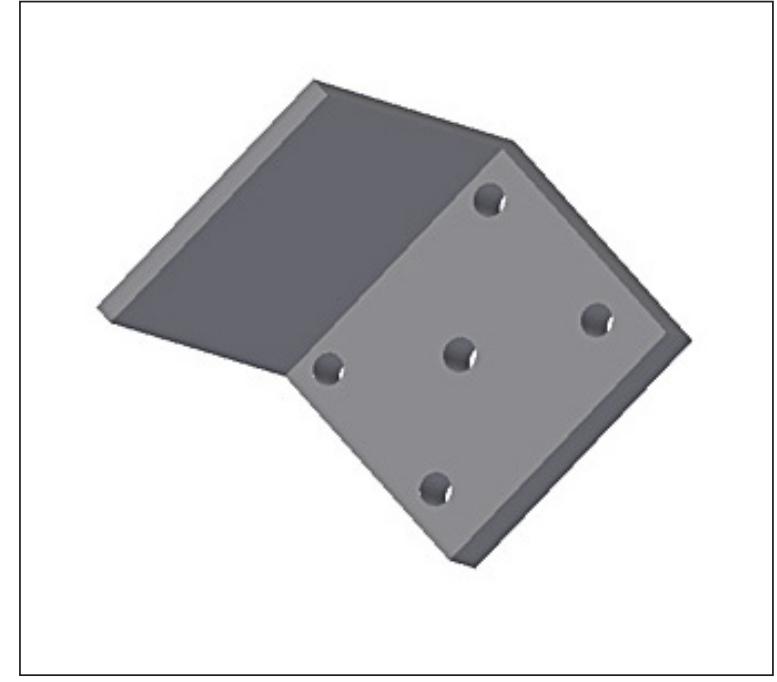

Fig. 5. Model of a two-sided connector

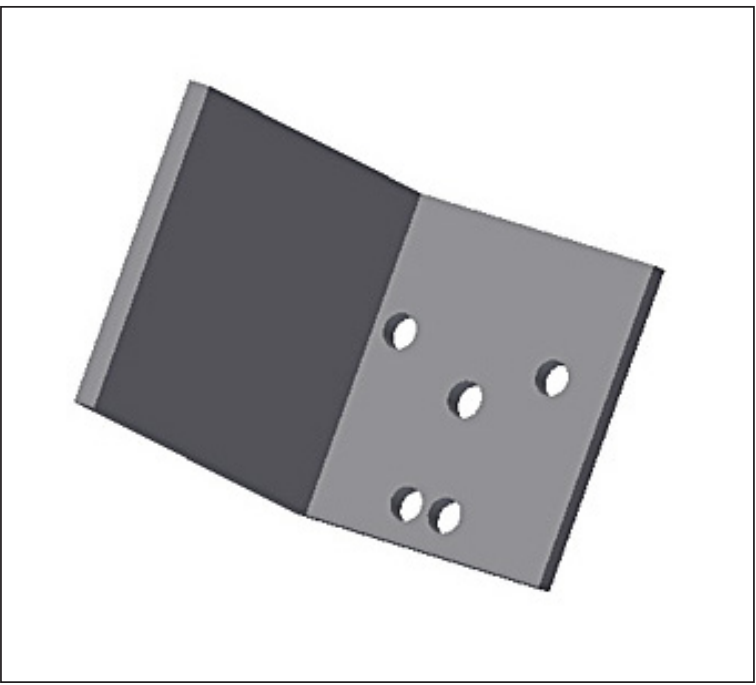

Fig. 6. Modifying the location of holes on the connector

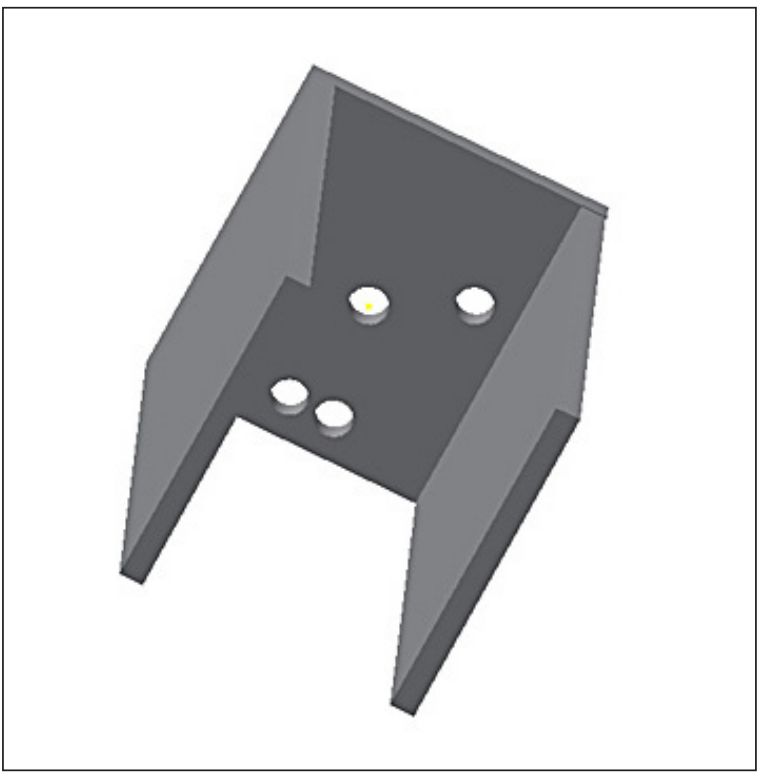

Fig. 7. Creating a model of a three-sided connector 


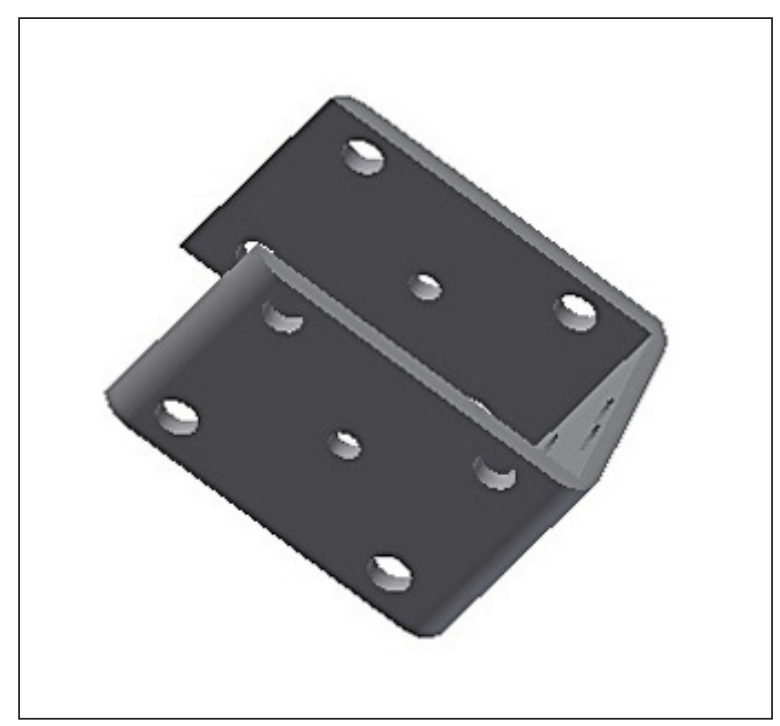

Fig. 8. Model of a three-sided connector with rounded corners

Figure 7 illustrates the process of designing a three-sided connector. The corners of the section are rounded in this model (Fig. 8). It is also possible to thread the holes.

\section{CONCLUSIONS}

The presented example of object parameterisation in Autodesk Inventor 2015 illustrates the possibility of quickly designing various types of structural connectors needed for a particular wooden or steel structure. The process of design involves editing a 2D drawing of a given shape. 3D models of a variety of connectors can be generated from a single drawing by applying appropriate parametric and geometric constraints. We were able to employ the parameterisation functionality of Inventor software as an efficient and time-effective method of designing specific connector's details.

\section{REFERENCES}

1. Brunetti G., Golob B. A feature-based approach towards an integrated product model including conceptual design information. Computer-Aided Design, 32(14), 2000, 877-887.

2. Cohn D. Evolution of computer-aided design. Desktop Engineering Digital Edition 2010. (Access online http://www.deskeng.com/de/evolutionof-computer-aided-design/)

3. Jaskulski A. AutoCAD 2015. The course of parametric and a nonparametric design. PWN, Warsaw 2014.

4. Morrical K. Start capturing reality: What every structural engineer needs to know. Reality Computing 2014. (Access online http://lineshapespace. com/start-capturing-reality-for-structural-engineers/)

5. Płuciennik P., Design elements of machines using the Autodesk Inventor program (in Polish). PWN, Warsaw 2013.

6. Pratt M.J. Anderson B.D., Ranger T. Towards the standardized exchange of parameterized featurebased CAD models. Computer-Aided Design, 37(12), 2005, 1251-1265.

7. Randy S. Parametric modeling with Autodesk Inventor 2014. SDC Publications, 2013.

8. Semotiuk L., Józwik J. Kuric I., Leleń M. Parameterisation of cutting technology programs in Heidenhain TNC 620 system (in Polish). Mechanik, No. 3, 2015, 178-186.

9. Sverko E. Drawing Autodesk Inventor standards with style. IMAGINiT Technologies 2015. (Access online http://www.cadalyst.com/)

10. Szal M, Herma S. The design processes effectiveness with Autodesk Inventor system (in Polish). Informatyczne Systemy Zarządzania, No. 2, 2011, 120-136.

11. Tremblay T., Inventor 2014 and Inventor LT 2014 essentials. Indianapolis 2013. 